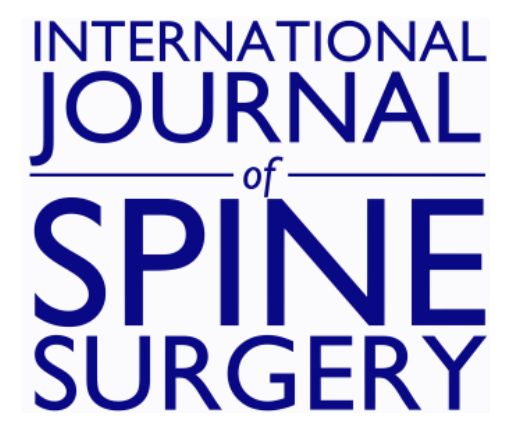

\title{
Outcomes of Demineralized Bone Matrix Enriched with Concentrated Bone Marrow Aspirate in Lumbar Fusion
}

Remi M. Ajiboye, Mark A. Eckardt, Jason T. Hamamoto, Benjamin Plotkin, Michael D. Daubs and Jeffrey C. Wang

Int J Spine Surg 2016, 10 ()

doi: https://doi.org/10.14444/3035

http://ijssurgery.com/content/10/35

This information is current as of April 25, 2023.

Email Alerts Receive free email-alerts when new articles cite this article. Sign up at:

http://ijssurgery.com/alerts

The International Journal of Sding Subrgerthttp://ijssurgery.com/ by guest on April 25, 2 2397 Waterbury Circle, Suite 1,

Aurora, IL 60504, Phone: +1-630-375-1432

(C) 2016 ISASS. All Rights Reserved. 


\section{Outcomes of Demineralized Bone Matrix Enriched with Concentrated Bone Marrow Aspirate in Lumbar Fusion}

Remi M. Ajiboye MD, ${ }^{1}$ Mark A. Eckardt BA, ${ }^{1}$ Jason T. Hamamoto BS, ${ }^{1}$ Benjamin Plotkin MD, ${ }^{1}$ Michael D. Daubs MD, ${ }^{2}$ Jeffrey C. Wang MD ${ }^{3}$

${ }^{1}$ UCLA Medical Center, Department of Orthopaedic Surgery, Santa Monica, CA 2University of Nevada School of Medicine, Department of Orthopaedic Surgery, Las Vegas, $N V^{3}$ Keck Medicine of USC, Department of Orthopaedic Surgery, Los Angeles, CA

\section{Abstract}

Background

Multiple studies have demonstrated that a significant amount of variability exists in various demineralized bone matrix (DBM) formulations, which casts doubts on its reliability in consistently promoting fusion. Bone marrow aspirate (BMA) is a cellular based graft that contains mesenchymal stem cells (MSCs) and growth factors can confer osteogenic and osteoinductive potential to DBM. The goal of this study was to describe the outcome of DBM enriched with concentrated BMA in patients undergoing combined lumbar interbody and posterolateral fusion.

Methods

Eighty patients with a minimum of 12 months of follow-up were evaluated. Fusion and rates of complication were evaluated. Functional outcomes were assessed based on the modified Odom's criteria. Multiple logistic regression analysis was used to examine the effects of independent variables on fusion outcome.

Results

The overall rate of solid fusion (i.e patients with both solid posterolateral and interbody fusion) was 81.3\% (65/80). Specifically, the radiographic evidence of solid posterolateral and interbody fusions were $81.3 \%(65 / 80)$ and $92.5 \%$ (74/80), respectively. Seven (8.75\%) patients developed hardware-related complications, 2 (2.5\%) patients developed a postoperative infection and $2(2.5 \%)$ patients developed clinical pseudarthrosis. Charlson comorbidity index (CCI) scores of 3 and 4 were associated with non-solid unions (CCI-3, p = 0.048; CCI-4, p = 0.03). Excellent or good outcomes were achieved in 58 (72.5\%) patients.

\section{Conclusions}

Patients undergoing lumbar fusion using an enriched bone graft containing concentrated BMA added to DBM can achieve successful fusion with relatively low complications and good functional outcomes. Despite these findings, more studies with higher level of evidence are needed to better understand the efficacy of this promising graft option.

KEYWORDS: LUMBAR FUSION; DEMINERALIZED BONE MATRIX; BONE MARROW ASPIRATE

\section{Introduction}

Transforaminal lumbar interbody fusion (TLIF) is often used in the management of spine pathologies including degenerative disk disease, deformity, trauma, severe instability, spondylolisthesis and pseudarthrosis. Several fusion aides are available to achieve a successful arthrodesis. These include autograft, allograft, bone graft enhancers, extenders and substitutes. Iliac crest bone graft (ICBG) offers good fusion success rates and remains the "gold standard". However, it is linked with certain potential morbidities (such as donor site pain, hematoma,

Downloaded from http://ijssurgery.com/ by guest on April 25, 2023 paresthesia and infection) that limit its use in spinal surgery. ${ }^{1}$ Demineralized bone matrix (DBM) is an osteoinductive allograft. It has been estimated that $93 \%$ of DBM consists of a weakly osteoconductive collagen scaffold, while the remainder of its components are osteoinductive factors such as bone morphogenetic proteins (BMPs) 2 and $7 .^{2}$ Multiple studies have reported variation between formulations of different DBM products as well as variation between lots of the same DBM products. ${ }^{3-5}$ This variability casts doubts on the reliability and efficacy of DBM in consistently promoting fusion. 
Bone marrow aspirate (BMA) is a cellular based graft that contains mesenchymal stem cells (MSC) and growth factors (such as BMPs 2 and 7) that can confer osteogenic and osteoinductive potential to osteoconductive scaffolds. ${ }^{6}$ BMA can be obtained via a relatively non-invasive method from the iliac crest during spine surgery and combined with DBM. The combination of BMA with DBM and allograft chips in a selective retention technology chamber has been shown to result in a graft matrix enriched with a 3- to 5 -fold increase in osteoprogenitor cells. ${ }^{7-10}$ Furthermore, the addition of osteoinductive factors derived from BMA to a graft material of DBM may negate the effects of unreliable concentrations of BMPs 2 and 7 in commercially available DBM products. ${ }^{6}$ To date, there is a dearth of literature describing the outcomes of DBM enriched with concentrated BMA in combined PLF and TLIF. Most of the published literature on outcomes of DBM has been described in patients undergoing anterior cervical discectomy and fusion (ACDF) and posterolateral lumbar fusion only.

The aim of this study was to describe the clinical and radiographic outcomes of an enriched graft material of concentrated BMA added to DBM/allograft chips in patients undergoing combined TLIF and PLF.

\section{Materials \& Methods}

After obtaining Institutional Review Board approval, a search of the medical record database for patients that underwent lumbar spine fusion by a single surgeon was performed for the period between May 2000 and January 2011. All patients in the study consented to surgery and the use of bone grafting.

Patients were included if they underwent a combined instrumented PLF and TLIF for the treatment of degenerative lumbar spine diseases with concomitant instability, if their procedure utilized concentrated BMA with DBM, and if there was at least 12 months of follow-up. Patients were excluded if they had a diagnosis of tumor, infection or fracture, underwent a posterolateral lumbar fusion alone, anterior lumbar interbody fusion, an uninstrumented fusion, or had less than 12 months of follow-up. Of the 157 patients that had combined PLF and TLIF, 73 were excluded because their surgery involved other bone graft options such as autograft, allograft, bone morphogenetic protein-2. Of remaining 84 patients that had PLF and TLIF using BMA with DBM, 4 were excluded for less than 12 months of follow-up, leaving a total of 80 patients for inclusion in this study.

Using the properties of an affinity column, bone marrow aspirates were processed with a selective cell retention system (CELLECT, DePuy Spine, Raynham, MA). ${ }^{11}$ Briefly, bone marrow aspirates were obtained from a single iliac crest using a 3-ported Jamshidi needle. Heparin was used in each syringe to inhibit clotting. In an effort to avoid dilution of the bone marrow with whole blood, $2 \mathrm{~mL}$ of bone marrow was obtained from each of the ports, and then the needle was advanced to new bone for additional draws of marrow. The total volume of bone marrow aspirates varied depending on the amount of graft material to be populated and was generally a ratio of 3 (aspirate volume):1 (graft material). The bone marrow was then run through the graft chamber of the selective cell retention device, which contained DBM and allograft chips, twice in accordance with the manufacturer's instructions. The enriched graft material was then placed in an interbody cage device and over the decorticated surfaces in the posterolateral gutters.

Information such as patient demographics, surgical and clinical data were obtained from the medical records. Demographic data included age and gender. Clinical data included smoking status, comorbidities, length of hospital stay, mean follow-up time, radiographic fusion grade, complications and functional outcomes. Co-morbidities were graded based on the Charlson co-morbidity index (CCI) scores. ${ }^{12}$ Smokers were defined as those who had continuously smoked for at least 1 year prior to surgery, as well as post-operatively. Surgical data included primary versus revision surgery, number of levels fused and estimated blood loss. Functional outcomes were assessed based on a 4-grade system: excellent, good, satisfactory and poor, in accordance with the modified Odom's criteria. ${ }^{13}$ Anteriorposterior (AP) and dynamic lateral radiographs were obtained in all patients as part of routine clinical follow-up. Posterolateral radiographic fusion was based on a 4-grade system, as defined by the Lenke 
classification: Grade A (definitely solid), Grade B (possibly solid), Grade C (probably not solid) and Grade D (definitely not solid). ${ }^{14}$ Interbody radiographic fusion was based on a 3-grade system as defined by the Brantigan, Steffee, Fraser (BSF): BSF-1 (radiographic pseudarthrosis), BSF-2 (radiographic locked pseudarthrosis) and BSF-3 (radiographic fusion).${ }^{15} \mathrm{~A}$ fellowship-trained and board-certified musculoskeletal radiologist graded fusion status based on radiographs. In cases of multilevel fusion with varying grades of fusion, the least fused segment was used to assign the final fusion grade. A solid fusion was defined as the concomitant presence of both Lenke A posterolateral and BSF-3 interbody fusion grades in the same patient (Figure 1). Radiographic pseudarthrosis was defined as the presence of Lenke $\mathrm{D}$ or BSF-1 fusion grade. The diagnosis of clinical pseudarthrosis was based on motion or non-union detected during revision surgery.

All statistical analyses were performed with R (version 3.0.2, R Foundation for Statistical Computing, Vienna, Austria). Fisher's exact test was used to assess differences in fusion outcomes based on single versus multilevel fusion. Multiple logistic regression analysis was used to examine the effects of variables such as patient age, gender, smoking, primary versus revision surgery, number of spine levels fused, length of hospital stay, length of follow-up, and Charlson comorbidity index score on fusion outcome. All analyses with a $p$ value of less than 0.05 were considered statistically significant.

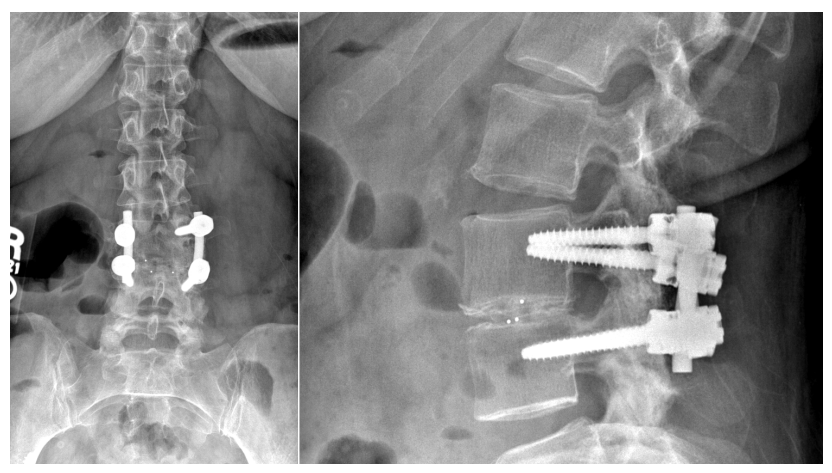

Fig. 1. Anterior-posterior and lateral radiograph showing solid posterolateral (Lenke A) and interbody (BSF-3) fusion in a 33 year-old female 1.5 years following lumbar 3-4 posterolateral and transforaminal lumbar interbody fusion using bone marrow aspirate/demineralized bone.

\section{Results}

\section{Demographic and clinical data}

The study group consisted of 80 patients ( 29 men and 51 women) with a mean age of 58.1 years (range $=16-80$ years) and a mean follow-up of 33.2 months (range $=12-89.6$ months) (Table 1$)$. The mean CCI score was 2.1 (range $=0-9$ ), the mean number of fused spine level was 1.3 (range $=1-3$ ), the mean estimated blood loss was $252.2 \mathrm{~mL}$ (range $=20-900 \mathrm{~mL}$ ) and the mean length of hospital stay was 5.2 days (range $=4-16$ days). 60 (76.3\%) patients were undergoing primary surgery and there were $8(10 \%)$ smokers (Table 1).

\section{Radiographic outcomes}

The overall rate of solid fusion (i.e patients with Lenke A and BSF-3 fusion grades) was 81.3\% (65/ 80). Specifically, the radiographic evidence of solid posterolateral (Lenke A) and interbody fusions (BSF-3) were 81.3\% (65/80) and 92.5\% (74/80), respectively (Table 2). In single-level fusions, solid posterolateral and interbody fusions were 79.7\% (47/ 59 ) and $93.2 \%$ (55/59), respectively. In multi-level fusions, solid posterolateral and interbody fusion were 85.7\% (18/21) and 90.5\% (19/21), respectively (Table 3).

\begin{tabular}{|l|r|}
\hline Table 1. Demographic, clinical and surgical variables. \\
\hline Mean age, SD* (range) & BMA (n= 80) \\
\hline Gender & $58.1+1-1.8(16-80)$ \\
\hline Male (\%) & $29(36.3 \%)$ \\
\hline Female (\%) & $51(63.7 \%)$ \\
\hline Mean Charlson co-morbidity index score (range) & $2.10(0-9)$ \\
\hline Smoker (\%) & $8(10 \%)$ \\
\hline Primary surgery (\%) & $60(75 \%)$ \\
\hline Mean estimated blood loss in milliliters (range) & $252.2(20-900)$ \\
\hline Mean number of fused spine levels (range) & $1.3(1-3)$ \\
\hline Mean number of hospital stay in days (range) & $5.2(4-16)$ \\
\hline Mean follow-up time in months (range) & $33.2(12-89.6)$ \\
\hline * SD (standard deviation). & \\
\hline
\end{tabular}


Using logistic regression analysis, CCI scores of 3 and 4 were associated with non-solid unions (CCI-3, CI: -7.28 to $-0.03, p=0.048$; CCI- 4, CI: -9.70 to $-0.46, p=0.03)$. None of the other variables were associated with non-solid unions (age, CI: -0.009 to 2.07, $\mathrm{p}=0.07$; gender, $\mathrm{CI}$ : -2.60 to $0.58, \mathrm{p}=0.21$; smoking, CI: -2.10 to $2.97, \mathrm{p}=0.73$; revision surgery, CI: -1.12 to $2.04, \mathrm{p}=0.57$; multilevel fusion, $\mathrm{CI}:-1.22$ to $2.56, \mathrm{p}=0.48$; length of hospital stay, $\mathrm{CI}$ : -0.50 to $0.63, \mathrm{p}=0.82$; length of follow-up time, $\mathrm{CI}:-0.02$ to $0.06, \mathrm{p}=0.34)$.

\section{Complications}

In the study group, 7 (8.75\%) patients developed hardware-related complications (in the form of hardware bursitis), 2 (2.5\%) patients developed a postoperative infection, 2 (2.5\%) patients developed pseudarthrosis and none of the patients developed

Table 2. Fusion grade.
\begin{tabular}{|l|r|}
\hline Overall fusion & BMA (n= 80) \\
\hline Both Grade A and BSF 3 & $65(81.3 \%)$ \\
\hline Posterolateral fusion & \\
\hline Grade A (\%) & $65(81.3 \%)$ \\
\hline Grade B (\%) & $5(6.3 \%)$ \\
\hline Grade C (\%) & $9(11.3 \%)$ \\
\hline Grade D (\%) & $1(1.3 \%)$ \\
\hline Interbody fusion & \\
\hline BSF-1 (\%) & $3(3.8 \%)$ \\
\hline BSF-2 (\%) & $3(3.8 \%)$ \\
\hline BSF-3 (\%) & $74(92.5 \%)$ \\
\hline
\end{tabular}

Table 3. Solid fusion (Lenke A and BSF-3) based on the number of fused levels.

\begin{tabular}{|l|r|r|}
\hline & BMA (n= 80) & p value \\
\hline Posterolateral fusion & & 0.75 \\
\hline Single-level (\%) & $47 / 59(79.7 \%)$ & \\
\hline Multi-level (\%) & $18 / 21(85.7 \%)$ & \\
\hline Interbody fusion & & 0.65 \\
\hline Single-level (\%) & $55 / 59(93.2 \%)$ & \\
\hline Multi-level (\%) & $19 / 21(90.5 \%)$ & \\
\hline
\end{tabular}

graft donor-site morbidities (Table 4).

\section{Functional outcomes}

Using the modified Odom's criteria, excellent or good results were achieved in 58 (72.5\%) patients in the study group (Table 5). Solid fusion was achieved in $44 / 58(75.9 \%)$ of patients with excellent or good outcomes and 21/22 (95.4\%) of patients with satisfactory or poor compared to $(\mathrm{p}=0.06)$.

\section{Discussion}

Combined PLF and TLIF techniques have traditionally used ICBG as the gold standard for achieving successful fusion. Due to the potential morbidities (such as donor site pain, paresthesia, hematoma, and infection) of ICBG harvest and limited graft supply in revision and multilevel fusion surgeries, there is continued interest in the spine surgery community for alternative bone graft options to aide with spine fusion. ${ }^{1}$ In this study, we found that patients undergoing combined TLIF and PLF using an enriched graft material containing concentrated BMA and DBM can achieve successful fusion with relatively low complications and good functional outcomes. To date, this is the largest study to report on the outcome of this graft option in patients undergoing combined PLF and TLIF.

There is a dearth of evidence in the literature describing the outcomes of DBM with concentrated BMA in combined PLF and TLIF. Most of the published literature on outcomes of DBM has been de-

\begin{tabular}{|l|r|}
\hline Table 4. Complications. \\
\hline Hardware-related complications & $7(8.75 \%)$ \\
\hline Infection & $2(2.5 \%)$ \\
\hline Clinical pseudoarthrosis & $2(2.5 \%)$ \\
\hline Graft donor-site morbidity & - \\
\hline
\end{tabular}

Table 5. Functional outcomes by the modified Odom's criteria.

\begin{tabular}{|l|r|}
\hline Excellent or Good (\%) & BMA (n= 80) \\
\hline Satisfactory (\%) & $58(72.5 \%)$ \\
\hline Poor (\%) & $11(13.7 \%)$ \\
\hline
\end{tabular}


scribed in patients undergoing ACDF and lumbar PLF only. In addition, only one study has been published that utilized DBM enriched with concentrated BMA. In the cervical spine, DBM with autograft was shown to achieve fusion rates ranging from $88.9 \%$ 97\% in patients undergoing anterior cervical discectomy and fusion (ACDF) ${ }^{16-18}$ In the lumbar spine, prospective controlled trials by Cammisa et al. and Sassard et al. reported disappointing fusion rates of $52 \%$ and $60 \%$, respectively in patients undergoing PLF alone with DBM and autograft. ${ }^{19,20}$ Conversely, other studies have described better success with DBM and autograft in the lumbar spine with fusion rates ranging from $70 \%$ - $91 \%$ in patients undergoing PLF alone. To the best of our knowledge, only two studies in the English literature have been published describing the outcomes of DBM in combined PLF and TLIF. In a comparative study by Ahn et al., DBM with autograft was compared with autograft alone in patients undergoing combined PLF and TLIF. ${ }^{21}$ At two years of follow-up, fusion success was $86 \%$ in patients in the DBM and autograft group compared to $87 \%$ in the autograft alone group. In a similar study by our group examining the outcome of DBM with BMA in combined PLF and TLIF in elderly patients ( $\geq 65$ years of age), the fusion success rate was $83.9 \%{ }^{22}$ The fusion success rate observed in both studies is comparable to the rate of $81.3 \% \mathrm{ob}-$ served in the current study.

There are several factors that are responsible for the failure to consistently achieve successful union with DBM in spinal fusions. As previously discussed, there is an unpredictable degree of variability both the concentrations of BMP among different lots of the same DBM formulation and the concentrations of BMP among different DBM formulations. Bae et al. showed that the variability of BMP concentrations among different lots of the same DBM formulation was higher than the variability of concentrations among those of different DBM formulations. ${ }^{5}$ In a separate in-vivo animal study by Bae et al., the variability of BMP concentrations in commercially available DBM products was noted to correlate with fusion success, which has been described to range from $0 \%$ to $75 \%{ }^{4}$ Additionally, the method of processing DBM and the type of carrier combined with DBM are also thought to contribute to the variability of
DBM in consistently promoting successful fusion. ${ }^{3,23,24}$ Lastly, the type of fusion construct may also play a role in fusion success with DBM. It is possible that the addition of an increased surface area for bone graft incorporation (in the form of an interbody fusion) contributes to a more stable construct required to promote the vascular ingrowth and cellular events needed to optimize the osteoinductive and osteogenic effects of concentrated bone marrow aspirate on bone fusion.

This current study is unique in that it utilizes a concentrated formulation of BMA to create an enriched graft material of DBM. It has been estimated that the proportion of osteogenic progenitors in bone marrow is between 1 in 5000 to 1 in 100,000 cells. ${ }^{25}$ During the process of bone marrow aspiration, it has been shown that the concentration of bone-marrow derived cells (including osteoprogenitors) in an aspirate is diluted rapidly by peripheral blood. ${ }^{26}$ The technique of concentrating BMA was developed as a practical means of delivering an increased number of osteogenic progenitor cells added to a graft site. The combination of BMA with DBM and allograft chips in a selective retention technology chamber has been shown to result in a graft matrix enriched with a 3- to 5-fold increase in osteoprogenitor cells. ${ }^{7-10}$ In a canine model of posterolateral lumbar fusions, Muschler et al. reported superior union rates with DBM enriched with concentrated BMA compared to DBM alone or DBM with non-concentrated BMA. ${ }^{10,27}$

The only patient characteristics noted to have an association with non-solid unions in this study were CCI scores of 3 and 4. CCI scores, which take into account patient age and the presence or absence of 19 comorbidities, was initially developed in 1987 to predict 1-year mortality after an inpatient hospitalization. ${ }^{28}$ It has since been validated across multiple medical and surgical sub-specialties. In the spine literature, CCI scores have been found to predict mortality, complications, and re-admissions after spine surgery ${ }^{29-31}$ It is not surprising that CCI scores are associated with decreased union rates in this study. For example, patients with multiple co-morbidities such as metabolic syndrome often have vascular diseases that may limit neo-angiogenesis and blood flow to a fusion site. In addition, advanced age, which is taken 
into consideration when generating a CCI score is also associated with poor bone quality, which may hinder fusion potential. ${ }^{32}$

Relatively low rates of complications were observed in this study. Implant-related complication in the form of hardware bursitis was the most common complication observed. Persistent low back pain despite a solid fusion in the absence of any obvious pain generator remains a challenging clinical scenario. In some of these patients, implant removal and exploration of fusion may be an appropriate option to alleviate their pain although this decision remains controversial due to the unknown mechanism of how implants generate pain.

Lastly, good or excellent results were achieved in the majority of patients in this study as measured by the modified Odom's criteria.

This study has several obvious limitations. Its retrospective design, possible selection bias associated with patient follow-up, and lack of a comparative group limits the generalizability of the results. Randomized control trials would be required to make more definitive conclusions about fusion rates, complications and functional outcomes of DBM enriched with concentrated BMA. In addition, cellular analysis of the bone marrow aspirate was not conducted on each patient. As a result, it is not possible to quantify the amount of osteoprogenitor cells in each aspirate. Another major limitation of this study is the method of radiographic assessment of fusion status. CT scan has been demonstrated to be more sensitive that radiographs for assessing fusion following instrumented lumbar fusion. ${ }^{33,34}$ Despite these limitations, this study is the largest of its kind and it provides valuable information and insight on a promising bone graft option for spinal fusion.

\section{Conclusion}

In summary, we found that patients undergoing combined PLF and TLIF with DBM enriched with concentrated BMA can achieve successful fusion with relatively low complications and good functional outcomes. Given the concerning potential adverse effects associated with ICBG donor-site morbidity, concentrated BMA with DBM may be an appropriate bone graft option for patients undergoing combined posterolateral and interbody fusions for degenerative lumbar spine disease. Despite these findings, more clinical studies with higher level of evidence are needed to better understand the efficacy of this promising graft option.

\section{References}

1. Dimitriou R, Mataliotakis GI, Angoules AG, Kanakaris NK, Giannoudis PV. Complications following autologous bone graft harvesting from the iliac crest and using the RIA: a systematic review. Injury. 2011;42 Suppl 2:S3-15.

2. Aghdasi B, Montgomery SR, Daubs MD, Wang JC. A review of demineralized bone matrices for spinal fusion: the evidence for efficacy. The surgeon: journal of the Royal Colleges of Surgeons of Edinburgh and Ireland. 2013;11(1):39-48.

3. Wang JC, Alanay A, Mark D, et al. A comparison of commercially available demineralized bone matrix for spinal fusion. European spine journal : official publication of the European Spine Society, the European Spinal Deformity Society, and the European Section of the Cervical Spine Research Society.

2007;16(8):1233-1240.

4. Bae H, Zhao L, Zhu D, Kanim LE, Wang JC, Delamarter RB. Variability across ten production lots of a single demineralized bone matrix product. The Journal of bone and joint surgery. American volume. 2010;92(2):427-435.

5. Bae HW, Zhao L, Kanim LE, Wong P, Delamarter RB, Dawson EG. Intervariability and intravariability of bone morphogenetic proteins in commercially available demineralized bone matrix products. Spine. 2006;31(12):1299-1306; discussion 1307-1298.

6. Khashan M, Inoue S, Berven SH. Cell based therapies as compared to autologous bone grafts for spinal arthrodesis. Spine. 2013;38(21):1885-1891.

7. Youssef JA WJ, Lieberman IH, et al. Osteoprogenitor-Enriched Allograft in Lumbar Spinal Fusion: Preliminary Findings from a Two-year Prospective Multi-Center Study. Paper presented at: North American Spine Society 23rd Annual Meeting Pre-Course Section on Spine Biologics and Research: 
Clinical Usage in Human Papers; October 2008; Toronto, ON.

8. Kadiyala S KK, Attawaia M, et al. Rapid bone regeneration in femoral defects by an autologous osteoprogenitor cell concentrate prepared using an intraoperative selective cell retention technique. Transactions of the 49th Annual Meeting of the Orthopaedic Research Society; 2003; New Orleans, LA.

9. Wang JC, Youssef, J.A., Lieberman, I.H., Brodke, D.S., Haynesworth, S.E., Lauryssen, C., , Patel T. Selective Cell Retention Technology for Spinal Fusion. International Meeting on Advanced Spine Techniques; 2003; Rome, Italy.

10. Muschler GF, Matsukura Y, Nitto H, et al. Selective retention of bone marrow-derived cells to enhance spinal fusion. Clinical orthopaedics and related research. 2005(432):242-251.

11. Lee K, Goodman SB. Cell therapy for secondary osteonecrosis of the femoral condyles using the Cellect DBM System: a preliminary report. The Journal of arthroplasty. 2009;24(1):43-48.

12. Quan H, Li B, Couris CM, et al. Updating and validating the Charlson comorbidity index and score for risk adjustment in hospital discharge abstracts using data from 6 countries. American journal of epidemiology. 2011;173(6):676-682.

13. Kepler CK, Rihn JA, Radcliff KE, et al. Restoration of lordosis and disk height after single-level transforaminal lumbar interbody fusion. Orthopaedic surgery. 2012;4(1):15-20.

14. Lenke LG, Bridwell KH, Bullis D, Betz RR, Baldus C, Schoenecker PL. Results of in situ fusion for isthmic spondylolisthesis. Journal of spinal disorders. 1992;5(4):433-442.

15. Brantigan JW, Steffee AD. A carbon fiber implant to aid interbody lumbar fusion. Two-year clinical results in the first 26 patients. Spine.

1993;18(14):2106-2107.

16. Demircan MN, Kutlay AM, Colak A, et al. Multilevel cervical fusion without plates, screws or autogenous iliac crest bone graft. Journal of clinical neuroscience : official journal of the Neurosurgical Society of Australasia. 2007;14(8):723-728.

17. Moon HJ, Kim JH, Kim JH, Kwon TH, Chung HS, Park YK. The effects of anterior cervical discectomy and fusion with stand-alone cages at two contiguous levels on cervical alignment and outcomes.
Acta neurochirurgica. 2011;153(3):559-565.

18. Topuz K, Colak A, Kaya S, et al. Two-level contiguous cervical disc disease treated with peek cages packed with demineralized bone matrix: results of 3-year follow-up. European spine journal : official publication of the European Spine Society, the European Spinal Deformity Society, and the European Section of the Cervical Spine Research Society.

2009;18(2):238-243.

19. Sassard WR, Eidman DK, Gray PM, et al. Augmenting local bone with Grafton demineralized bone matrix for posterolateral lumbar spine fusion: avoiding second site autologous bone harvest. Orthopedics. 2000;23(10):1059-1064; discussion 1064-1055.

20. Cammisa FP, Jr., Lowery G, Garfin SR, et al. Two-year fusion rate equivalency between Grafton DBM gel and autograft in posterolateral spine fusion: a prospective controlled trial employing a side-byside comparison in the same patient. Spine. 2004;29(6):660-666.

21. Ahn DK, Moon SH, Kim TW, Boo KH, Hong SW. Demineralized bone matrix, as a graft enhancer of auto-local bone in posterior lumbar interbody fusion. Asian spine journal. 2014;8(2):129-137.

22. Ajiboye RM, Hamamoto JT, Eckardt MA, Wang JC. Clinical and radiographic outcomes of concentrated bone marrow aspirate with allograft and demineralized bone matrix for posterolateral and interbody lumbar fusion in elderly patients. European spine journal : official publication of the European Spine Society, the European Spinal Deformity Society, and the European Section of the Cervical Spine Research Society. 2015;24(11):2567-2572.

23. Lee YP, Jo M, Luna M, Chien B, Lieberman JR, Wang JC. The efficacy of different commercially available demineralized bone matrix substances in an athymic rat model. Journal of spinal disorders \& techniques. 2005;18(5):439-444.

24. Peterson B, Whang PG, Iglesias R, Wang JC, Lieberman JR. Osteoinductivity of commercially available demineralized bone matrix. Preparations in a spine fusion model. The Journal of bone and joint surgery. American volume. 2004;86-A(10):2243-2250. 25. Muschler GF, Midura RJ. Connective tissue progenitors: practical concepts for clinical applications. Clinical orthopaedics and related research. 2002(395):66-80. 
26. Muschler GF, Boehm C, Easley K. Aspiration to obtain osteoblast progenitor cells from human bone marrow: the influence of aspiration volume. The Journal of bone and joint surgery. American volume. 1997;79(11):1699-1709.

27. Muschler GF, Nitto $H$, Matsukura $Y$, et al. Spine fusion using cell matrix composites enriched in bone marrow-derived cells. Clinical orthopaedics and related research. 2003(407):102-118.

28. Charlson ME, Pompei P, Ales KL, MacKenzie $\mathrm{CR}$. A new method of classifying prognostic comorbidity in longitudinal studies: development and validation. Journal of chronic diseases.

1987;40(5):373-383.

29. Woods BI, Hohl JB, Braly B, Donaldson W, 3rd, Kang J, Lee JY. Mortality in elderly patients following operative and nonoperative management of odontoid fractures. Journal of spinal disorders \& techniques. 2014;27(6):321-326.

30. Voskuijl T, Hageman M, Ring D. Higher Charlson Comorbidity Index Scores are associated with readmission after orthopaedic surgery. Clinical orthopaedics and related research.

2014;472(5):1638-1644.

31. Whitmore RG, Stephen JH, Vernick C, et al. ASA grade and Charlson Comorbidity Index of spinal surgery patients: correlation with complications and societal costs. The spine journal : official journal of the North American Spine Society. 2014;14(1):31-38.

32. Okuda S, Oda T, Miyauchi A, Haku T, Yamamoto T, Iwasaki M. Surgical outcomes of posteri- or lumbar interbody fusion in elderly patients. The Journal of bone and joint surgery. American volume. 2006;88(12):2714-2720.

33. Santos ER, Goss DG, Morcom RK, Fraser RD. Radiologic assessment of interbody fusion using carbon fiber cages. Spine. 2003;28(10):997-1001.

34. Shah RR, Mohammed S, Saifuddin A, Taylor BA. Comparison of plain radiographs with CT scan to evaluate interbody fusion following the use of titanium interbody cages and transpedicular instrumentation. European spine journal : official publication of the European Spine Society, the European Spinal Deformity Society, and the European Section of the Cervical Spine Research Society. 2003;12(4):378-385.

\section{Disclosures \& COI}

No funds were received in support of this work. No relevant financial disclosures are associated with this work.

\section{Corresponding Author}

Remi M. Ajiboye, M.D. UCLA Department of Orthopaedic Surgery, 1250 16th Street, Suite 3142, Santa Monica, CA 90404. Remi.Ajiboye@gmail.com.

Published 17 October 2016.

This manuscript is generously published free of charge by ISASS, the International Society for the Advancement of Spine Surgery. Copyright @ 2016 ISASS. To see more or order reprints or permissions, see http://ijssurgery.com. 